\title{
Mathematical Modeling of The SIP Call
}

\author{
Ravonimanantsoa N.M., Randriamitantsoa A.A, Rakotondraina T.E
}

\begin{abstract}
The use of VoIP is becoming more common in the world of the Internet

Modeling of voice over IP at the server is an interesting work for future uses of this technology. The reason for this mathematical modeling is to forecast or predict the levels of bandwidth allocated for a number of user. While using different parameters assumed to be used according to their use in the server ie CPU, NIC, memory. The allocation of new parameters, such as the protocol and are used logiciles recently studied. In this paper, we propose a new mathematical model polynomial models to predict the use of the network interfaces using the SIP protocol. Our method aims to determine the number of clients possible for a mini VoIP server. It has several advantages such as the definition of a minimum required for the deployment of a VoIP family and also the limited number of clients using the server capacity. For our experiment we conducted a simulation of SIP call and deduce the mathematical model by interpolation.

Voip,Sip,Asterisk,polynom
\end{abstract}

\section{INTRODUCTION}

This template, the use of the Internet is widely seen as a tool in our daily life, different research has been conducted to improve the user satisfaction on this technology in full bloom.

However, in recent research, it is reported that there is a kind phenomenon of ourselves to the "all IP". This phenomenon marks the beginning of a new era in the field of telecommunications, having crossed for centuries in the resale switched telephony, we are in the era of Telephony IP. This new deal does not mean that the switched telephone networks are more efficient but the reason lies in the idea of cost for infrastructure.

The convergence of traditional telephony to IP telephony is an area of research that deserves many attentions.

Much research has been done on this subject since its appearance [1]. Here we focus on SIP will be the future of IP telephony [2] for its performance.

To conduct our research we have done experiments and simulation.

In this paper, we propose an approach based on experimentation to build a polynomial of degree three (3) for the behavior of the SIP call over a network. We built an experiment with a VoIP server Asterisk running on a Debian 6.0 operating system and two X-lite softphone client that is installed on a Windows XP operating systems to collect data simulation. Using these data, we construct a polynomial model describing the evolution of the amount of captured package. The behavior of each instance during the call is also analyzed.
The motivation of this paper is organized as follows in Section 2. The experimental, results of experiments and observations are described in Section 3. Section 4 analyzes the behavior of our curve. A polynomial model is constructed in Section 5.and finally the conclusion and future work of our research..

\section{MOTIVATION}

\section{Speech Based Dialog Query System over Asterisk PBX Server[3]}

Research entitled "Speech Dialog System based query over Asterisk PBX server" gives insight into the theoretical details and implementation of a speech-based query system for dialogue on the Asterisk server. Asterisk PBX server can be connected to traditional analog phones or PSTN trunk lines (Public Switched Telephone Network) for connection with the PCI Special equipment and VOIP phones using different VoIP protocols such as SIP, IAX and H.323. This system is designed for queries Railway Enquiry in Indian (Hindi) and English. this document describes how a query system can be deployed Asterisk server to two analog phones and softphones as client interfaces and the results are analyzed recognition accuracy.

From this paper, we have tested the idea of the reality of a call using SIP.

\section{THE EXPERIENCE}

In this section we will talk briefly about the hardware and software used in our experiment, then in a second part we will talk about the experience itself.

\section{The architecture}

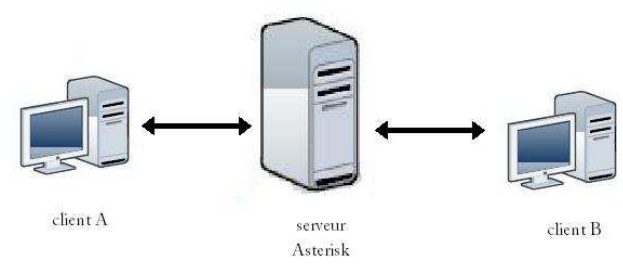

Figure 1. Architecture 
Our network architecture is minimized by a server connected to two client machines to make the call (minimum). All this is connected is connected by a switch

\section{Experience}

- $\quad$ First, we begin to initiate the call from SIP softphone client windows. Next, we move to a packet capture on our Asterisk server via the command "tcpdump"

- Next, we arrange the packets captured by the command "tcpstat" so it can be traced by our gnuplot graph plotter.

- Finally, we observe the behavior of our graph for a definite time before the call is hung up.

\section{Result}

The results give us the following figures

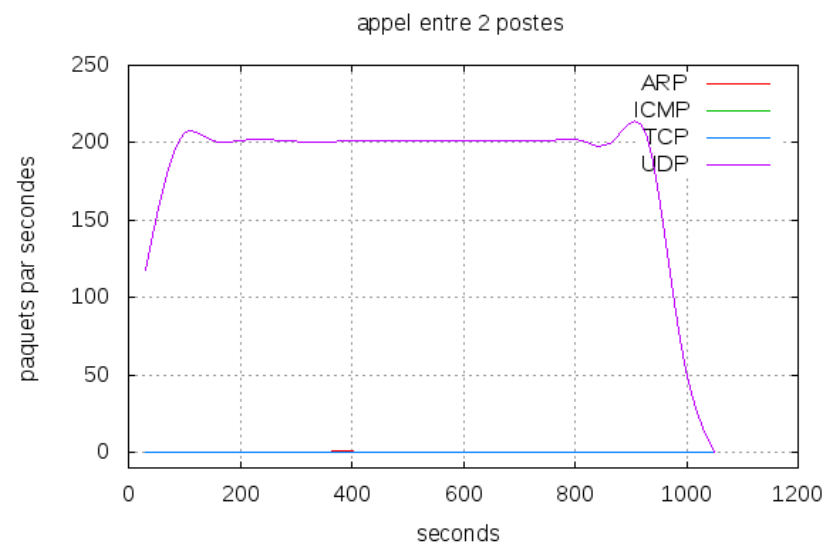

Figure 2. Graphe of SIP call

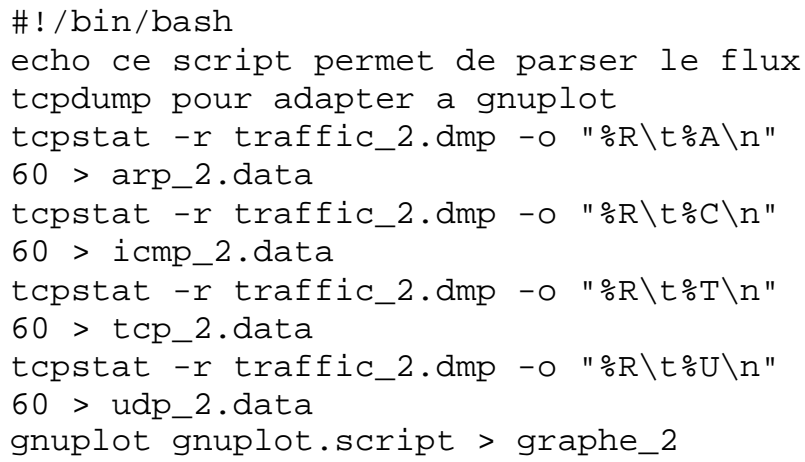

the shell script tcpstat enabled us to plot the graph is below

Figure 3. tcpstat shell

\section{Wia: $\operatorname{SIP} /$}

14:53:15.036004 IP debian.local.sip >

192.168.1.113.21460: SIP, length: 500

E...C.@.-i..o...q.S...>SIP/2.0 100 Trying

Via: SIP/2.0/UDP 192.168.1.1

14:53:15.036535 IP debian.local.sip >

192.168.1.222.44094: SIP, length: 944

E...\{...@............ gINVITE

$\operatorname{sip} 2222 @ 192.168 .1 .222: 44094$; rinstance $=0 \mathrm{c}$

14:53:15.036616 IP debian.local.sip >

192.168.1.113.21460: SIP, length: 516

E...D.@.-X...o.....S....NSIP/2.0 180 Ringing

Via: SIP/2.0/UDP 192.168.1.

Figure 4. Tcpdump extract

\section{ANALYSIS OF THIS CURVE}

Based on our findings, we noticed that during the initiation of the call or SIP opens the session with "Guest" we observe a peak in the number of packets per second, this peak is shown at the closing the session can be found or the message "BYE".

So we can conclude that:

- Sends the message invites a lot of packet from the conversation

- Similarly for "bye"

- Finally, our simulation shows that an unanswered call may cause overload our network interface by this peak

\section{POLYNOMIAL MODEL}

After we obtained our graph was exported in the Matlab software has come for us to deduce a polynomial model with our graph using the function of matlab polyfit. We have chosen three (3) as the degree of our polynomial by the fact that we define three parameters for our research includes: memory, processor and speed of our networks. Thus we have obtained the following result

$$
\begin{aligned}
& \text { > plot }(\mathrm{x}, \mathrm{y}) \\
& >>\mathrm{p}=\text { polyfit }(\mathrm{x}, \mathrm{y}, 3) \\
& \mathrm{p}= \\
& 1.0 \mathrm{e}+004 * \\
& -0.0000 \quad 0.0000 \quad-0.0005 \quad 1.0358
\end{aligned}
$$

Figure 5. Matlab script and result 


\section{REFERENCES}

the polynomial will be of this form

$\left.P(x)=A x^{3}+B x^{2}+C x+D\right)$

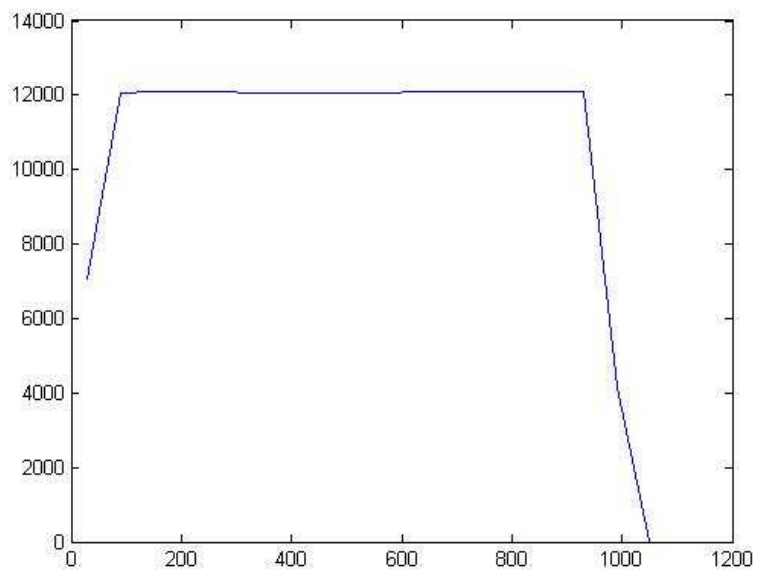

Figure 6. Matlab curv

With

$\mathrm{A}=$ parameter of memory

$\mathrm{B}=$ parameter of processor

$\mathrm{C}=$ Parameter of network

And D the constant of our sip status

\section{CONCLUSION AND FUTURE WORK}

In this paper it is shown that the introduction to the call for a SIP is a peak which is not significant but may cause a malfunction of the interface resale after several attempts if $n$ 'is not picked up at the Asterisk server.

Experimental results show that the communication itself represents a number of this package increases with number of calls. Our simulation and our experience leads us to conclude that establish a SIP session is a certain level of packet-level network interface and a number of session afraid to challenge the level of our communication network interface. Finally, we conclude that we can model our call by determining the constants A, B, C and D to see what type of computer for any number of client for the Asterisk server. We anticipate that the design of such a model requires the simulation with several poste. Diverses methods can lead us in this endeavor to improve the efficiency of our model..
[1] Saurabh Goel,Muhua Bhattacharya,speech based dialog query system over Asterisk PBX Server,ICSPS 2010

[2] H. Abdelnur, R. State, I. Chrisment, and C. Popi, "Assessing the security of VoIP Services," in Integrated Network anagement, IM 2007. 10th IFIP/IEEE International Symposium on Integrated Network Management, Munich, Germany, 21-25. IEEE, May 2007, pp. 373-382.

[3] I E. Chen, "Detecting dos attacks on sip systems," VoIP Management and Security, 2006. 1st IEEE Workshop on, pp. 53-58, April $2006 .$.

[4] D. Geneiatakis, T. Dagiuklas, G. Kambourakis, C. Lambrinoudakis,S. Gritzalis, K. Ehlert, and D. Sisalem, "Survey of security vulnerabilities in session initiation protocol," Communications Surveys \& Tutorials, IEEE, vol. 8, no. 3, pp. 68-81, Qtr. 2006..

[5] A. Habib, M. M. Hefeeda, and B. K. Bhargava, "Detecting service violations and dos attacks," in In Proceedings of 2003 Internet Society Symposium on Network and Distributed System Security (NDSS03, 2003, pp. 177-189.M. Young, The Technical Writer's Handbook. Mill Valley, CA: University Science, 1989.

[6] P. montoro, E. casilari, A comparative stutdy of VoIP standarts with asterisk,Fourth International conference on Digital Télécommunications 2009

[7] Ravonimanantsoa N M.V,Randriamitantsoa P.A "Comparison Of The Consumption Of Resources Between HTTP And SIP" Advanced Engineering Forum Volume 1 p330

Ravonimanantsoa N.Manda-vy

Student at University of Antananarivo and have thesis in computer application in telecommunication, born may 23, 1978 in Antsirabe (Madagascar), have a diploma computer engineer in 2008 at University of Fianarantsoa(Madagascar)and mathematic degree in 2002 at University of Antananarivo(Madagascar.

He has two paper published in international journal and teach at Ecole Supérieur Polytechnique d'Antananarivo 